\title{
Role of Intravascular Ultrasound in Prediction of Acute Side Branch Occlusion in Coronary Artery Bifurcation Lesions Just After Provisional Stenting
}

\author{
Ahmed Mohamed Hamed ${ }^{1 *}$ MSc; Ayman Kamal Abo Elmagd ${ }^{1}$ MD; Mostafa Ibrahim Mokarrab ${ }^{1}$ MD;
} Ahmed Mahmoud Sabry ${ }^{2}$ MD

*Corresponding Author:

Ahmed Mohamed Hamed ahmedsalha73@gmail.com

Received for publication january 10,2021;Accepted january 31, 2021;

Published online january 31, 2021.

$$
\begin{aligned}
& \text { Copyright } 2021 \text { The Authors } \\
& \text { published by Al-Azhar } \\
& \text { University, Faculty of Medicine, } \\
& \text { Cairo, Egypt. All rights reserved. } \\
& \text { This an open-access article } \\
& \text { distributed under the legal terms, } \\
& \text { where it is permissible to } \\
& \text { download and share the work } \\
& \text { provided it is properly cited. The } \\
& \text { work cannot be changed in } \\
& \text { anyway or used commercially. } \\
& \text { doi: 10.21608/aimj.2021.55264.1384 } \\
& { }^{1} \text { Cardiology Department, Faculty of } \\
& \text { medicine, Al-Azhar university , } \\
& \text { Cairo, Egypt. } \\
& { }^{2} \text { Cardiology Department, Military } \\
& \text { Medical Academy, Egypt. }
\end{aligned}
$$

\begin{abstract}
Background: Coronary bifurcation lesion is important subject in percutaneous coronary intervention (PCI). While much progress has been made in PCI facilities, bifurcation stenting is still has a lot of problems.

Objective: The aim of this study was the usefulness of intravascular ultrasound (IVUS) to help in the prediction of occlusion of side branch (SB) after stenting the main vessel (MV) in bifurcation lesions.

Patients and methods: The period of this study was from May 2019 to December 2020, and included 80 patients who were submitted for elective coronary angiography to the cardiology department at Maadi Military Hospital, and showed bifurcation lesions treated with provisional stenting using IVUS measurements.

Results: This study included two groups according to the occlusion of SB after main vessel stenting. The 1st group had the patients with occlusion of SB ( $n=18)$ and the 2nd group had the patients without occlusion of SB ( $n=62)$. The thinner plaque of main vessel (MV) at junction position with SB, MV plaque area and diameter ratio of SB were the factors which could predict acute occlusion of SB after provisional stenting.

Conclusion: In coronary bifurcation lesions, we can use intravascular ultrasound (IVUS) to help in the prediction of occlusion of SB after provisional stenting, and these predictors can help the operators to prepare the best strategy for treating bifurcation lesions.
\end{abstract}

Keywords: Provisional Stenting; Bifurcation Lesion; Intravascular Ultrasound.

Disclosure: The authors have no financial interest to declare in relation to the content of this article. The Article Processing Charge was paid for by the authors.

Authorship: All authors have a substantial contribution to the article.

\section{INTRODUCTION}

The European Bifurcation Club (EBC) describes coronary bifurcation lesions as coronary artery stenosis with more than $50 \%$ stenosis of the main vessel (MV) arising beside to or affecting the root of side branches ${ }^{\mathbf{1 , 2}}$. The most important factor in bifurcation stenosis is to make the stenting technique as straightforward as possible by using the provisional single stent strategy ${ }^{3}$. This technique is based on data from many studies which did not show any advantage of two-stent over one-stent strategy ${ }^{4}$. Intravascular ultrasound (IVUS) is an important tool during PCI as it provides data about the properties of pre-PCI lesions as vulnerable plaques, lesion severity, length, morphology, stent expansion, apposition, and complications after stent implantation ${ }^{5,6}$. Several studies compared findings with angiographic evaluation alone versus under the guidance of IVUS, and showed lower major adverse cardiac events (MACE) and cardiac death rates at follow up were seen in the IVUS group ${ }^{7,8,9}$. At the beginning of the procedure, risk factors for $\mathrm{SB}$ occlusion after provisional stenting technique should be recognized such as SB stenosis and acute angel of $\mathrm{SB}^{\mathbf{1 0 - 1 5}}$. In this study, we are trying to understand the usefulness of IVUS to help in the prediction of SB collapse after provisional stenting.

\section{PATIENTS AND METHODS}

This was a prospective observational study performed from May 2019 to December 2020, and included 80 patients submitted for elective percutaneous coronary intervention at the cardiology department in Maadi Military Hospital. Patients with recurrent chest pain and their coronary angiography revealed bifurcation stenosis stented by provisional stenting were included in the inclusion criteria. We excluded the patients presented for primary PCI, patients with left main bifurcation stenosis and patients with side branch had a TIMI flow $\leq 2$ before MV stenting. The institutional ethics committee approved the research procedure. Venous samples were withdrawn upon admission, then collected into 
standardized tubes containing the potassium ethylene di nitro tetra acetic acid (EDTA) reagent, and stored at room temperature. Serum troponin, creatinine, lipid profile were included. ECG and echocardiography were performed. Coronary angiography plus PCI data were recorded, and IVUS measurements were taken. The Volcano Eagle Eye IVUS Platinum Catheter was used in this study, and was inserted into the main vessel (MV) in all patients prior to stent implantation. After IVUS measurements, the definition of SB was the branch with diameter $\geq 1.5 \mathrm{~mm}$. SB was considered to be occluded if it had TIMI flow $\leq 2$ after main vessel stenting. The IVUS catheter crossed the lesion and withdrawn at about $1 \mathrm{~mm} / \mathrm{sec}$ after guide wire crossing in the MV to show the imaging sequence, and it began distal to the stenosis site, and terminated at the aorta. We calculated the minimum lumen diameter (MLD) of $\mathrm{MV}$, minimal luminal area (MLA), minimum vessel diameter (MVD), and external elastic membrane (EEM) area of MV at the junction site. The plaque area was determined as EEM CSA minus MLA. We measured the thickness of the MV plaque at the junction position, and we used the thinner plaque at the junction position as a measure of the quantity of plaque. The plaque type was fatty, fibrous, mixed, and calcified plaque. We calculated total and luminal diameter of SB in the ostial site of SB. The total SB diameter was the length from media to opposite media, and the luminal SB diameter was the length from intima to opposite intima. We specified the ratio of SB diameter as the total diameter of SB divided on luminal diameter of SB. We had a good stent size (diameter and length) with the aid of IVUS, and we measured the stent area after stenting to confirm stent apposition and expansion. SB pre dilatation prior to MV stent was left to the decision of the operator.

Results were subjected to the analysis using Statistical Program for Social Science (SPSS) software version 18.0 (SPSS Inc., Chicago, IL). We considered frequency and percentage as the qualitative results. We considered mean \pm standard deviation (SD) as the quantitative results. T-test of significance was used when we compared between two means. Chi-square (X2) test of significance was used when we compared the ratio between two qualitative parameters. To classify the factors which predicted the occlusion of SB after MV stenting, we used univariate and multivariate logistic-regression analysis. To evaluate the cut-off value, sensitivity and specificity of predictors of occlusion of the SB after stenting of $\mathrm{MV}$, the receiver operating characteristics (ROC) curve was used. $\mathrm{P}$ value $\leq 0.05$ indicated that the result was statistically significant

RESULTS

\begin{tabular}{|c|c|c|c|c|c|c|}
\hline \multirow{2}{*}{\multicolumn{2}{|c|}{ Demographic data }} & Non SB occluded group & SB occluded group & \multirow{3}{*}{ Test value } & \multirow{3}{*}{ P-value } & \multirow{2}{*}{ Sig. } \\
\hline & & No. $=62$ & No. $=18$ & & & \\
\hline Sex & $\begin{array}{c}\text { Females } \\
\text { Males }\end{array}$ & $\begin{array}{l}16(25.8 \%) \\
46(74.2 \%)\end{array}$ & $\begin{array}{c}3(16.7 \%) \\
15(83.3 \%)\end{array}$ & & & NS \\
\hline Age & $\begin{array}{c}\text { Mean } \pm \text { SD } \\
\text { Range }\end{array}$ & $\begin{array}{c}55.48 \pm 7.26 \\
39-68\end{array}$ & $\begin{array}{c}56.00 \pm 7.30 \\
43-67\end{array}$ & $-0.265 \bullet$ & 0.792 & NS \\
\hline HTN & $\begin{array}{l}\text { No } \\
\text { Yes }\end{array}$ & $\begin{array}{l}17(27.4 \%) \\
45(72.6 \%)\end{array}$ & $\begin{array}{c}6(33.3 \%) \\
12(66.7 \%)\end{array}$ & $0.238 *$ & 0.626 & NS \\
\hline Smoking & $\begin{array}{l}\text { No } \\
\text { Yes } \\
\end{array}$ & $\begin{array}{l}29(46.8 \%) \\
33(53.2 \%)\end{array}$ & $\begin{array}{c}8(44.4 \%) \\
10(55.6 \%) \\
\end{array}$ & $0.030^{*}$ & 0.861 & NS \\
\hline DM & $\begin{array}{l}\text { No } \\
\text { Yes }\end{array}$ & $\begin{array}{l}33(53.2 \%) \\
29(46.8 \%)\end{array}$ & $\begin{array}{c}11(61.1 \%) \\
7(38.9 \%)\end{array}$ & $0.350 *$ & 0.554 & NS \\
\hline
\end{tabular}

Table 1: Comparison between the demographic data and risk factors among the study groups

The patients in this study divided into two groups according to the occlusion of SB after MV stenting into SB occluded group which represented $22.5 \%$ of the patients $(n=18)$ and non SB occluded group which represented $77.5 \%$ of the patients $(n=62)$. In non SB occluded group, males represented $74.2 \%(n=46)$ and mean age was 55.48 \pm 7.26. In SB occluded group males represented $83.3 \%(n=15)$ and mean age was $56.00 \pm 7.30$. Demographic data and risk factors among the study groups were presented in table1. 


\begin{tabular}{|c|c|c|c|c|c|c|c|c|}
\hline & \multicolumn{4}{|c|}{ Univariate } & \multicolumn{4}{|c|}{ Multivariate } \\
\hline & \multirow{2}{*}{ P-value } & \multirow{2}{*}{ OR } & \multicolumn{2}{|c|}{ 95\% C.I. } & \multirow{2}{*}{ P-value } & \multirow{2}{*}{ OR } & \multicolumn{2}{|c|}{ 95\% C.I. } \\
\hline & & & Lower & Upper & & & Lower & Upper \\
\hline Thinner MV plaque & 0.001 & 241.778 & 17.248 & 3389.147 & 0.001 & 494.976 & 14.743 & 16618.665 \\
\hline MV MLD & 0.265 & 0.634 & 0.285 & 1.412 & - & - & - & - \\
\hline MV MVD & 0.615 & 1.349 & 0.420 & 4.329 & - & - & - & - \\
\hline MV lumen CSA & 0.145 & 0.854 & 0.692 & 1.056 & - & - & - & - \\
\hline MV EEM CSA & 0.759 & 1.024 & 0.882 & 1.188 & - & - & - & - \\
\hline MV plaque area & 0.001 & 2.313 & 1.417 & 3.775 & 0.003 & 2.957 & 1.439 & 6.079 \\
\hline SB total diameter & 0.930 & 1.052 & 0.339 & 3.260 & - & - & - & - \\
\hline SB luminal diameter & 0.004 & 0.194 & 0.064 & 0.591 & 0.813 & 0.769 & 0.087 & 6.794 \\
\hline SB diameter ratio & 0.001 & 3.261 & 1.637 & 6.498 & 0.034 & 5.140 & 1.136 & 23.261 \\
\hline
\end{tabular}

Table 2: Univariate and multivariate logistic regression analysis to the predictors of SB occlusion

As regard demographic data, risk factors, laboratory investigations, ECG changes and PCI data, no statistically significant difference was found between the study groups as p-values were more than 0.05 . As regard IVUS measurements, the thinner plaque of MV at junction position with SB, MV plaque area and diameter ratio of SB were the factors which could predict acute occlusion of SB after provisional stenting by the aid of multivariate logistic regression analysis, as statistically significant difference was found between the study groups as p-values were $0.001,0.003$ and 0.034 respectively (table2).

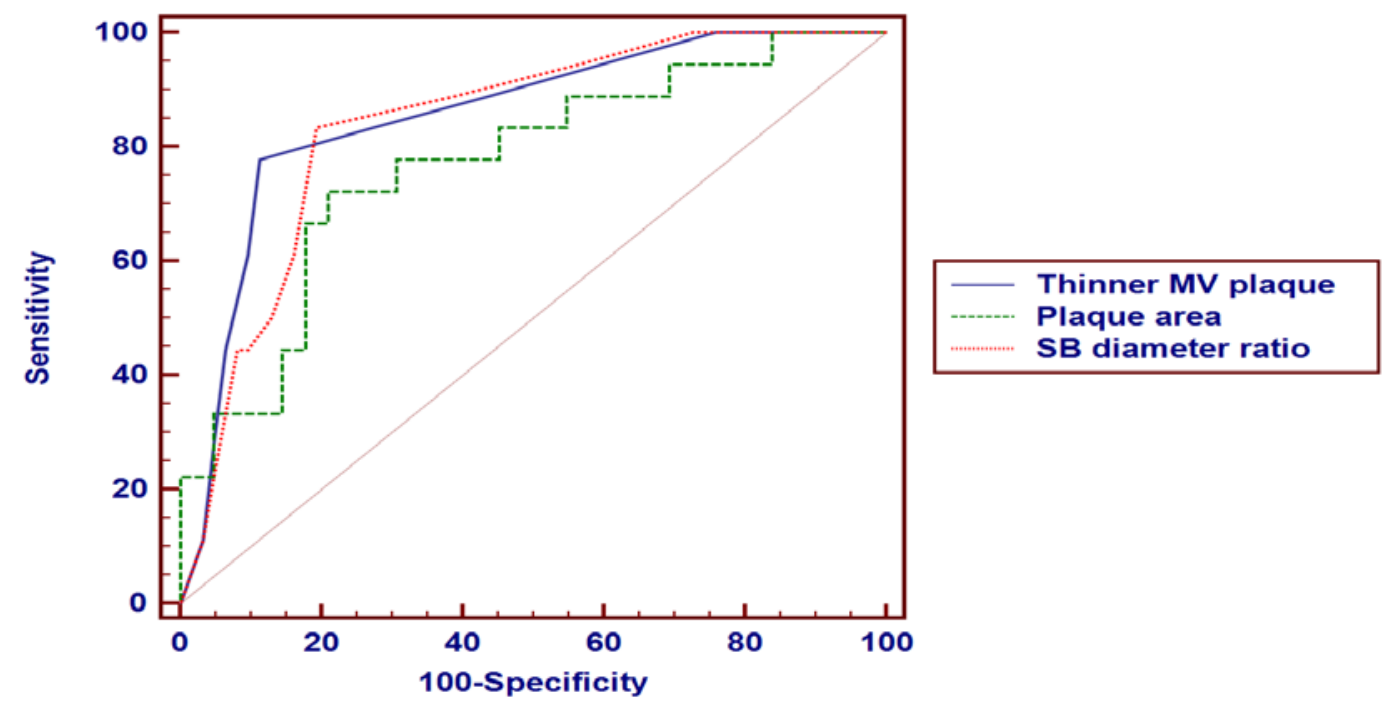

Fig.1: ROC curve for predictors of SB occluded group

Receiver Operating Characteristic (ROC) Analysis showed that $85 \%$ was the accuracy of thinner MV plaque at junction position with SB by $77.7 \%$ sensitivity and $88.7 \%$ specificity, at cut off predictive value of occlusion of the SB after provisional stenting $>0.8$ to discriminate between the study groups. The accuracy of MV plaque area was $77 \%$, by $72.2 \%$ sensitivity and $79 \%$ specificity, at cut off predictive value of occlusion of the SB after stenting of MV $>11.25$ to discriminate between the study groups. The accuracy of SB diameter ratio was $83 \%$, by $83.3 \%$ sensitivity and $80.6 \%$ specificity, at cut off predictive value of occlusion of the SB after stenting of MV $>1.4$ to discriminate between the study groups (Fig1). 


\section{DISCUSSION}

This study was done to demonstrate the usefulness of IVUS to help in the prediction of SB collapse after provisional stenting in patients who presented for elective PCI. Patients were divided into group with SB occlusion $(\mathrm{n}=18)$ and group without SB occlusion $(n=62)$. Patients in each group were matched to the other group regarding demographic data, clinical examination, laboratory investigations, PCI data, and IVUS measurements. As regard demographic data, there was no statistically significant difference among the study groups as regard age ( $\mathrm{P}: 0.792)$ and sex ( $\mathrm{P}: 0.422)$, and agreed with this result a study done by Dong et al. ${ }^{16}$ on 1,171 patients, but it disagreed with our study as they divided the study to high and low bifurcation angel groups, and the group with high angel demonstrated higher percentage of SB collapse $(63 / 600,10.5 \%)$ than the low angle group $(25 / 600,4.2 \%)(\mathrm{P}<0.001)$. As regard risk factors, no statistically significant difference was found among the study groups as regard HTN (P : 0.626), smoking (P : 0.861) and DM (P : 0.554), and agreed with this result a study done by Yang et al. ${ }^{17}$ on 207 patients presented for elective PCI with the aid of optical coherence tomography (OCT). As regard PCI data, no statistically significant difference was found among the study groups regarding target $\mathrm{SB}, \mathrm{SB}$ pre dilatation, stent size and diameter as $\mathrm{P}$ values were $>0.05$, and agreed with this result a study done by Nobuo et al. ${ }^{18}$ on 174 patients with the aid of IVUS measurements before stenting. Our study included the patients who were presented for elective PCI due to the risk of thrombus dislodgement and no reflow by IVUS catheter in case of primary PCI, and agreed with us a study done by Yang et al. ${ }^{17}$ due to the risk of OCT catheter to cause the same complication, but disagreed with us a study done by Valerie et al. ${ }^{19}$ on 174 patients who underwent primary PCI, and the percentage of SB occlusion was $22.5 \%$, and the factors which could predict acute occlusion of SB after provisional stenting were non-LAD culprit artery, larger area of myocardial infarction, and adequate collaterals, but they depended only on coronary angiography without using IVUS. In our study, the factors which predicted the occlusion of SB after provisional stenting were the thinner plaque of $\mathrm{MV}$ at the junction position with SB, plaque area in MV and diameter ratio of $\mathrm{SB}$, and agreed with this result a study done by Nobuo et al. ${ }^{18}$, which demonstrated that the thinner plaque in $\mathrm{MV}$ and diameter ratio of SB were the factors which predicted the occlusion of SB after provisional stenting as $\mathrm{p}$ values were less than 0.05 , but MV plaque area was not a predictor of occlusion of SB after MV stenting as $\mathrm{p}$ value was 0.96 . In our study, SB diameter ratio could predict occlusion of SB after MV stenting, but SB diameter stenosis could predict occlusion of SB after MV stenting in a study done by Kefei et al. ${ }^{20}$ on 1,601 bifurcation lesions and treated with a provisional stenting. Our study did not include SB lesion length as a factor could predict the occlusion of SB after MV stenting as IVUS catheter introduced only in $\mathrm{MV}$, but this role appeared in a study done by Mohsin et al. ${ }^{15}$ on 200 patients, and showed that SB lesions were longer in the group with SB occlusion than the group without
SB occlusion (4.66 \pm 3.11 and $3.53 \pm 0.51$, p value $<0.001)$. Our factors which could predict acute occlusion of SB after MV stenting depended on IVUS data, but there was a study depended on CT coronary angiography and was done by Jin Joo et $\mathrm{al}^{21}$ on 65 patients. Our study had a high percentage (22.5\%) of occlusion of SB after MV stenting as we included SB with small diameter $\geq 1.5 \mathrm{~mm}$, and there were other studies had similar percentage as Jin Joo et al. ${ }^{22}$, Nobuo et al. ${ }^{18}$, and Valerie et al. ${ }^{19}$ which showed percentage of SB occlusion just after provisional stenting as $18 \%, 19.1 \%$ and $22.5 \%$ respectively. There were a lot of studies searched the factors which predicted the occlusion of SB after provisional stenting, and they depended only on the coronary angiography data, but limited studies, including our study, used the IVUS measurements for this purpose.

\section{CONCLUSION}

In coronary bifurcation lesions, we can use intravascular ultrasound (IVUS) to help in the prediction of SB occlusion after provisional stenting, and these predictors can help the operators to prepare the best strategy for treating bifurcation lesions.

\section{REFERENCES}

1. Louvard Y, Medina A., et al. Definitions and classifications of bifurcation lesions and treatment. $J$ Euro Intervention.2015; 11 (SupplV):V23-26. DOI: 10.4244/EIJV11SVA5.

2. Lassen JF, Holm NR, Banning A, et al. Percutaneous coronary intervention for coronary bifurcation disease: 11th consensus document from the European Bifurcation Club. Euro Intervention .2016; 12:38-46. DOI: 10.4244/EIJV12I1A7.

3. Banning AP, Lassen JF, Burzotta F, et al. Percutaneous coronary intervention for obstructive bifurcation lesions: the 14th consensus document from the European bifurcation club. Euro Intervention. 2019; 15(1):90-8. DOI: 15(1):90-8.

4. Sawaya FJ, Lefèvre $T$, Chevalier $B$, et al. Contemporary approach to coronary bifurcation lesion treatment. J Am Coll Cardiol Intv. 2016; 9(18):1861-78. DOI: 10.1016/j.jcin.2016.06.056.

5. Koskinas KC, Ughi GJ, Windecker S, et al. Intracoronary imaging of coronary atherosclerosis: validation for diagnosis, prognosis and treatment. Eur Heart J. 2016; 37:524-535a-c.DOI: 10.1093/eurheartj/ehv642.

6. Toutouzas K, Benetos G, Karanasos A, et al. Vulnerable plaque imaging: updates on new patho biological mechanisms. Eur Heart J. 2015; 36: 3147-54. DOI: 10.1093/eurheartj/ehv508.

7. Chen L, Xu T, Xue XJ, et al. Intravascular ultrasound guided drug-eluting stent implantation is associated with improved clinical outcomes in patients with unstable angina and complex coronary artery true bifurcation lesions. International Journal of Cardiovascular Imaging. 2018; 34(11):1685-96. DOI: 10.1007/s10554-018-1393-2. 
8. Elgendy IY, Mahmoud AN, Elgendy AY, et al. Outcomes with intravascular ultrasound-guided stent implantation: a meta analysis of randomized trials in the era of drug eluting stents. Circ Cardiovasc Interv.2016;;9:e003700.

DOI:10.1161/CIRCINTERVENTIONS.116.003700.

9. Bavishi C, Sardar P, Chatterjee S, et al. Intravascular ultrasound-guided vs angiography guided drugeluting stent implantation in complex coronary lesions: meta-analysis of randomized trials. $\mathrm{Am}$ Heart J. 2017; 185:26-34. DOI: 10.1016/j.ahj.2016.10.008.

10. Zhang D, Xu B, Yin D, Li YP,He Y, You SJ, et al. Clinical and angiographic predictors of major side branch occlusion after main vessel stenting in coronary bifurcation lesions. Chin Med J. 2015; 128(11):1471-8. DOI: 10.4103/0366-6999.157654 .

11. Lee SH, Lee JM, Song YB, Park TK, Yang JH, Hahn JY, et al. Prediction of side branch occlusions in percutaneous coronary interventions by coronary computed tomography: the CT bifurcation score as a novel tool for predicting intra procedural side branch occlusion. EuroIntervention. 2019;15(9):e78895.Doi: 10.4244/EIJ-D-18-00113.

12. Bai J, Yue Y, Feng HQ, Hao SX, Peng L, Zhang M, et al. Impact of main vessel calcification on procedural and clinical outcomes of bifurcation lesion undergoing provisional single-stenting intervention: a multi center, prospective, observational study. $J$ Geriatr Cardiol. 2019;16(2):156-63.DOI: $\quad$ 10.11909/j.issn.16715411.2019.02.012.

13. Palinggi BP, Firman D, et al. Carina bifurcation angle and side branch occlusion in coronary bifurcation lesions intervention: angiographic lesions characteristic role in determining its relation. Int $J$ Angiol. 2019; 28(02):137-41. DOI: 10.1055/s-00381676042.

14. Zhao Z, Zhang D, Xu B, Wang H, Gao G, Yin D, et al. Is side branch lesion length an independent predictor of acute side branch occlusion in provisional strategy? Analysis of 524 consecutive bifurcation lesions. Catheter Cardiovasc Interv. 2018; 91:599-607. DOI: 10.1002/ccd.27497.
15. Mohsin M, Hamid Khan, Muhammad Saleem, et al. Assessment of Predictors of Side Branch Occlusion after Main Vessel Stenting in Coronary Bifurcation Lesions in Patients Undergoing Percutaneous Coronary Intervention. $J$ Coll Physicians Surg Pak.2018; 741-7.

16. Dong Zhang, Bo Xu, Dong Yin, et al. How Bifurcation Angle Impacts the Fate of Side Branch After Main Vessel Stenting. Catheterization and Cardiovascular Interventions. 2015; 85:706-15. DOI: $10.1002 /$ ccd.25858.

17. Yang Cao, Gary Mintz, Mitsuaki Matsumura et al. The relation between optical coherence tomographydetected layered pattern and acute side branch occlusion after provisional stenting of coronary bifurcation lesions. Car rev.2018; 122-31. https://doi.org/10.1016/j.

18. Nobuo Sakamoto, Yasuto Hoshino, Hiroyuki Mizukami, et al. Intravascular Ultrasound Predictors of Acute Side Branch Occlusion in Coronary Artery Bifurcation Lesions Just After Single Stent Crossover. Catheterization and Cardiovascular Interventions. 2016; 87:243-50.

19. Valerie khoo, Liang Shen, Vanessa Khoo, et al. incidence and predictors of side branch compromise in primary percutaneous coronary intervention for acute myocardial infarction. J invasive cardiol. 2014; 26(7):297-302.

20. Kefei Dou, Dong Zhang, Bo Xu, et al. An Angiographic Tool for Risk Prediction of Side Branch Occlusion in Coronary Bifurcation Intervention The RESOLVE Score System (Risk prEdiction of Side branch OccLusion in coronary bifurcation intervention). JAAC and Cardiovascular Interventions. 2015; VOL 8,NO. 1, 39-46.

21. Jin Joo Park, Eun Ju Chun, Young-Seok Cho, et al. Potential Predictors of Side-Branch Occlusion in Bifurcation Lesions after Percutaneous Coronary Intervention: A Coronary CT Angiography Study. Radiology. 2014; Vol 271: Number 3.2.55-65. 